OPEN ACCESS

Edited by:

Lutz Jäncke,

University of Zurich, Switzerland

Reviewed by:

Ramesh Kandimalla,

Emory University, USA

Jingwen Niu,

University of Pennsylvania, USA

*Correspondence:

Hokyoung Ryu

hryu@hanyang.ac.kr

Received: 01 April 2016 Accepted: 27 May 2016 Published: 13 June 2016

Citation: Jeong E and Ryu H (2016) Melodic Contour Identification Reflects the Cognitive Threshold of Aging. Front. Aging Neurosci. 8:134. doi: 10.3389/fnagi.2016.00134

\section{Melodic Contour Identification Reflects the Cognitive Threshold of Aging}

\author{
Eunju Jeong and Hokyoung Ryu* \\ Department of Arts and Technology, Hanyang University, Seoul, South Korea
}

Cognitive decline is a natural phenomenon of aging. Although there exists a consensus that sensitivity to acoustic features of music is associated with such decline, no solid evidence has yet shown that structural elements and contexts of music explain this loss of cognitive performance. This study examined the extent and the type of cognitive decline that is related to the contour identification task (CIT) using tones with different pitches (i.e., melodic contours). Both younger and older adult groups participated in the CIT given in three listening conditions (i.e., focused, selective, and alternating). Behavioral data (accuracy and response times) and hemodynamic reactions were measured using functional near-infrared spectroscopy (fNIRS). Our findings showed cognitive declines in the older adult group but with a subtle difference from the younger adult group. The accuracy of the melodic CITs given in the target-like distraction task (CIT2) was significantly lower than that in the environmental noise (CIT1) condition in the older adult group, indicating that CIT2 may be a benchmark test for age-specific cognitive decline. The fNIRS findings also agreed with this interpretation, revealing significant increases in oxygenated hemoglobin (oxyHb) concentration in the younger ( $p<0.05$ for $\Delta$ pre - on task; $p<0.01$ for $\Delta$ on - post task) rather than the older adult group (n.s for $\Delta$ pre - on task; n.s for $\Delta$ on - post task). We further concluded that the $\mathrm{oxyHb}$ difference was present in the brain regions near the right dorsolateral prefrontal cortex. Taken together, these findings suggest that CIT2 (i.e., the melodic contour task in the target-like distraction) is an optimized task that could indicate the degree and type of age-related cognitive decline.

Keywords: contour identification task, melodic contours, cognitive decline, aging, dorsolateral prefrontal cortex, hemodynamic responses

\section{INTRODUCTION}

Aging is believed to shift our attentional frame of reference more inwardly. Both seeing and hearing respond to such changes (Faubert, 2002; Parbery-Clark et al., 2011), and a certain level of withdrawal in cognitive functions is inevitable (Deary et al., 2009; De Oliveira et al., 2014). As the life expectancy increases, the loss of perceptual acuity and cognitive skills together cause significant difficulties in the elderly's independence, such as communicating with others.

Speech in noise (SIN) perception, for example, requires an active interplay between auditory and cognitive capacities (Shinn-Cunningham and Best, 2008; Parbery-Clark et al., 2009a,b; Francis, 2010) and is one of the frequent difficulties that arises among older adults. When a speech stream 
is presented in a fairly quiet environment, attention to target speech is relatively easy. However, as the environmental noise, which contains meaningful sounds or utterances, becomes louder or competes against the speech stream. SIN perception therefore requires more effort and attentional resources (Pichora-Fuller, 2003, 2006; Heinrich et al., 2008; Russo and Pichora-Fuller, 2008). In other words, a higher level of auditory cognition, such as sound segregation and selective attention-the ability to separate important, relevant sound streams from extraneous ones-is necessary. In this respect, auditory scene analysis (ASA, Bregman, 1999) is likely intricately involved.

Many neurological studies suggest that older adults have a lower level of activation in the auditory cortex compared to younger adults (Ostroff et al., 2003; Snyder and Alain, 2005). In contrast, their cognitive-related cortices are highly activated. Notably, activation in cognitive areas other than the perceptual areas indicates an important compensatory mechanism for aging. This interpretation has been demonstrated in previous studies (e.g., Alain et al., 2009; Wong et al., 2009), which have reported that the elderly displays a more diffuse network involving the frontal and ventral brain regions, while younger people tend to show a more streamlined cortical network of auditory regions in response to spoken word processing in a noisy environment.

Taken together, these findings suggest an interactive contribution of the two factors in aging: the perceptual acuity of the target sound and the cognitive strategy to effectively cope with the target sound as well as the noise. Research, however, has failed to identify the contributions of each factor. Therefore, the present study presents the diverse acoustic features of music in an empirical study and examines the cognitive strategies and perceptual acuity used by both older and younger adults to cope with the exogenous complexity of the acoustic features of music (in particular, melodic contour) in this sense.

Music can be a methodologically time-efficient tool to evaluate cognitive changes that occur with aging. Notably, a pattern perception of music (i.e., melodic contour identification) often requires discrete pitch perception. Hearing loss and temporal resolution problems are generally observed in a pitch discrimination task (Tremblay et al., 2003), and an aging effect on cognitive functioning can also be seen in the neural substrates and their connectivity to musical stimuli (Bones and Plack, 2015).

Melodic contours share some common features with verbal speech and are the relative changes in pitch that are typically described as "rising," "falling," or "stationary." As shown in Figure 1, different types of contours mimic prosodic information that can convey both non-verbal and subtle emotional aspects of speech (Inspector et al., 2013). In particular, previous researchers have found that contours and timbres are primarily processed at the brain stem level (Schirmer and Kotz, 2006; Leitman et al., 2009) and are the most noticeable compared to other structural elements of music across all age groups and cultures (Thompson and Schellenberg, 2006).

Previous studies have also suggested that melodic contours could be used as assessment stimuli for perceptual and cognitive dysfunctions. For example, a clinical application has been applied for cochlear implant (CI) problems (Galvin et al., 2008, 2009; Loui et al., 2008; Luo et al., 2014a,b). The general findings were that contour recognition and timbre perception are key in dictating auditory impairment. Even more interesting, Zhu et al. (2011) examined the effects of pitch, timbre, and time difference in the identification of simultaneous contours, and they reported that timbre and time differences were the most important features for the identification task. Therefore, these two features provide perceptual cues for an auditory stream and allow for segregation of the target auditory stimuli in a multi-layered auditory environment.

That being said, several empirical studies have used melodic contours to show cognitive decline in patient populations that have included those with traumatic brain injury and mild cognitive impairment (Belleville et al., 2011; Jeong and Lesiuk, 2011; Rahman et al., 2011; Jeong, 2013). Notably, a recent neurophysiological study (Jeong and Ryu, 2016) found that cognitive loads increase when distracting/competing sounds are present in the target sound identification task.

A note with regard to the melodic contour identification task (CIT) is further needed here. Both music and speech perception are types of time-based information processing and are subserved by the capacity of temporal processing and time resolution (Bones et al., 2014). In addition, up and down patterns in pitch contour are similar to the prosodies of human speech, which convey individuals' emotional tones (Inspector et al., 2013).

These shared features between music and speech are processed in overlapping areas in the brain and in the medial prefrontal region in particular (Schirmer and Kotz, 2006; Patel, 2008; Fedorenko et al., 2009). This area modulates the junction between cognition and emotion and is inwardly directed toward mental states, including introspective thoughts about the self, autobiographical memory, and familiarity (Kelley et al., 2002; Platel et al., 2003; Gilbert et al., 2005; Ochsner and Gross, 2005), as well as tonal information of melodic contours (Platel et al., 2003; Janata, 2005; Wittfoth et al., 2010). The prefrontal region remains relatively intact until the final stage of dementia (Cuddy and Duffin, 2005), which suggests the possibility that it can be sensitive assessment stimuli to detect age-related cognitive impairments of aging.

Further, music processing assimilates real-world ASA. Generally, more than two streams (i.e., a melody and accompaniment or two melodies) are present in a musical scene. Likewise, in our auditory environment, multi-layered sound streams (e.g., voices, environmental sounds, and additional noises) are simultaneously present. The instrumental timbre provides a perceptual cue to segregate and/or integrate multi-layered streams of music, while voice timbre plays the same role in conversation against other environmental noises and/or among a crowd (Broadbent, 1958; Bregman, 1999). That is, one can employ diverse and subtle acoustic features, such as loudness, pitch, and timbre, to differentiate the target sound from the distracting sounds (Allen et al., 2011; Best et al., 2011; Luo et al., 2014a,b). Previous research (Janata, 2005; Jeong and Lesiuk, 2011) has claimed that, when given in a polyphonic texture, the melodic CIT can mimic the nature of real-world auditory surroundings in which environmental sounds, speech and music are simultaneously heard. For this study, the identification of 


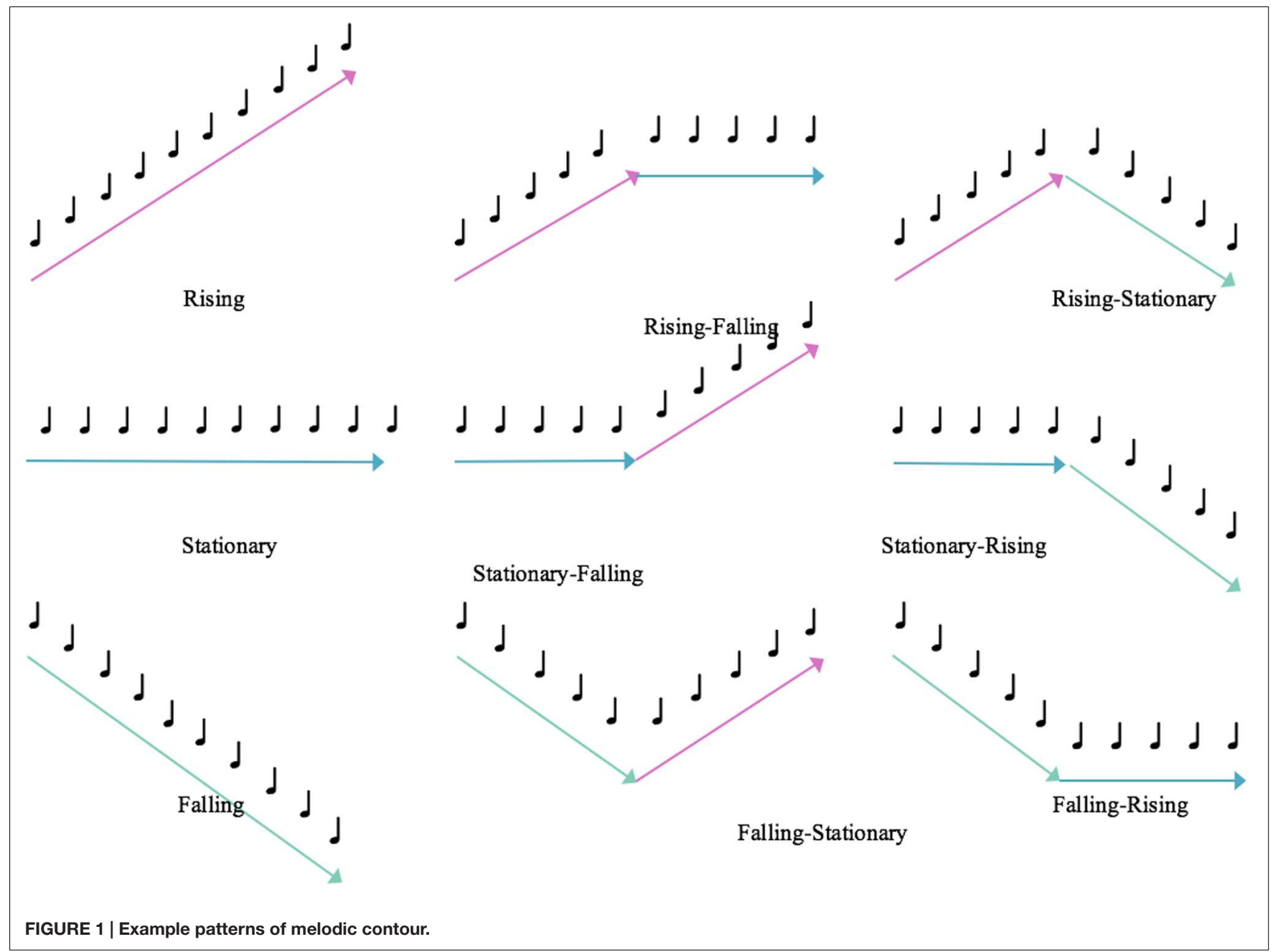

the two melodic contours is thus considered as a representative experimental task.

It can be seen that timbre, in particular, is the primary cue for segregating concurrent melodic contours. The role of timbre in music scene analysis has also been well supported by neurological evidence (Satoh et al., 2001; Janata et al., 2002). In segregation, a salient or familiar timbre would be quickly processed, and the less salient or unfamiliar timbre might require a shift in attention. In this sense, selective attention is also examined. Hence, given that timbre discrimination is highly associated with speech perception in aging (Zhu et al., 2011; Grassi and Borella, 2013; Arehart et al., 2014), timbre discrimination and selective attention can sufficiently indicate age-related communication skills.

Taken together, these results indicate that the identification of melodic contour(s) given in a single- or multi-layered context represents one's ability to segregate sounds and apply selective attention, while also imposing varying levels of cognitive load. The present study thus examines the behavioral and neurophysiological responses to the melodic CIT in various conditions and compares the difference between young and older adults in performance of the melodic CIT.

\section{MATERIALS AND METHODS}

\section{Participants}

Thirteen college students (male $=10$, female $=3$ ) and 14 older adults (male $=7$, female $=7$ ) were voluntarily recruited via a web advertisement ${ }^{1}$. None of the participants were professionally trained in music and had a neurological medical history or sensory impairment. The mean age was $23.54(S D=1.66)$ years for the younger adults and $56.07(S D=6.35)$ years for the older adults. The average education level was 12.0 years for the younger adults and 12.43 years for the older adults with a non-significant difference between groups $(t=-0.461$, n.s.). All participants were right-handed, as indicated by the Edinburgh Handedness Inventory (Oldfield, 1971).

\section{Apparatus}

\section{Music Stimuli (Experimental Sound Sources)}

The six contour stimuli were a combination of three contours (ascending, descending, and staying the same) adopted from Jeong (2013). Each contour consists of five tones presented at different frequencies as follows: (1) 262, 392, 440, 494, and 


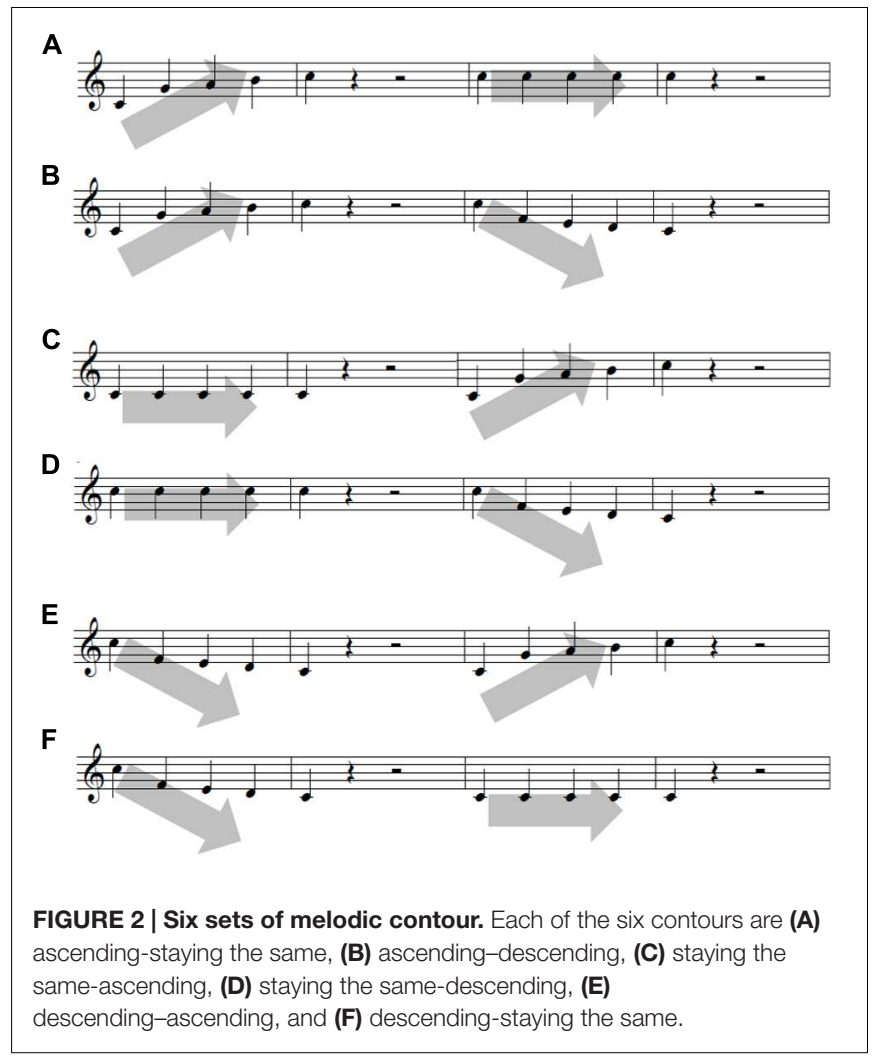

$523 \mathrm{~Hz}$ for an ascending contour, (2) repetition of a single tone of $392 \mathrm{~Hz}$ for a staying the same contour, and (3) 523, 349, 330, 294, and $262 \mathrm{~Hz}$ for a descending contour. The contours were then combined in the following sets (Figure 2). The contours were played by one of three synthesized instruments (for timbre control: piano, flute, or string), and their amplitudes were identically normalized. The six contours in conjunction with the three instruments produced a total of 18 contour sets. All were generated using an MIDI synthesizer (YAMAHA DGX 230) connected to a Logic Pro $X$, and the experimental apparatus was implemented on a computer containing Visual Studio.

\section{Contour Identification Task}

Table 1 shows how the contours were combined in each of the three CITs. For CIT1, environmental sounds (e.g., raining, crying, laughing, babbling, and applause) were presented with the contours as a control condition. In contrast, in both CIT2 and CIT3, two different contour sets were presented at the same time using different instruments. In CIT2, participants were asked to selectively attend to a contour that was played by a specific instrument, which was cued on the computer monitor. For instance, two musical contours were played simultaneously by both the piano and the flute, and participants had to selectively attend to the flute as the target contour (Figure 3). CIT3 was more complex, and the participants were asked to shift their attention from one contour to another as the instruments displayed on the computer screen were changed. For example, the two sound contours were simultaneously presented by both the piano and the flute, and the indicator
TABLE 1 | Structure of melodic contour identification task.

\begin{tabular}{lccc}
\hline & \multicolumn{3}{c}{ Type of listening tasks } \\
\cline { 2 - 4 } Sound presented & Focused & Selective & Alternating \\
\hline $\begin{array}{l}\text { Melodic contour with } \\
\text { environmental sounds }\end{array}$ & ClT1 & - & - \\
$\begin{array}{l}\text { Melodic contour with } \\
\text { target-like distraction }\end{array}$ & - & CIT2 & CIT3 \\
\hline
\end{tabular}

on the computer screen was changed from a piano (on the first contour) to a flute (for the second contour); participants were asked to separately identify the contour created by each instrument. For all CITs, the participants chosed the contour by clicking the arrows on the computer monitor (Figure 3).

\section{Measures}

\section{Hemodynamic Responses}

$\mathrm{OxyHb}$ was measured to evaluate the participants' cognitive activation and loads imposed by the given tasks (Peck et al., 2013; Sato et al., 2013; Ogawa et al., 2014; Yasumura et al., 2014). For this evaluation, functional near-infrared spectroscopy (fNIRS) and non-invasive monitoring of cortical tissue oxygenation (changes in both oxyHb and deoxygenated hemoglobin, $\mathrm{HHb}$ ) during cognitive, motor, and sensory stimulation (Jobsis, 1977; Ferrari and Quaresima, 2012) were employed. We used a 16-channel Spectratech OEG-16 (Shimadzu Co. Ltd., Kyoto, Japan) (Figure 4). The task-related hemodynamic changes in amount of oxyHb were recorded in 16 channels with a sampling rate of $0.65 \mathrm{~s}$. In addition to the fNIRS data, we collected behavioral data, including task performance accuracy and reaction time.

\section{Procedure}

This study was approved by the Institutional Review Board of Hanyang University (HYI-14-127-3). Participants were voluntarily recruited via a web advertisement ${ }^{1}$. Prior to the experiment, all participants gave written informed consent in accordance with the Declaration of Helsinki.

On the day of the experiment, participants were administered the study instructions, and completed a demographic questionnaire. Then, a band-type NIRS containing an array of 12 probes was attached to each participant's forehead. The probes were connected to the main board of the NIRS, which communicated with a computer. The melodic contour tasks were delivered via headphones with volume control; visual cues specifying the target musical stimulus were presented to the participants on a monitor (LG LED 24MA53D). A 20-s baseline was presented before, within, and after three CITs while the participants fixed their eyes on the center of the monitor. Prior to the main experimental sessions, the participants had practiced demonstrated a greater than $80 \%$ accuracy in the CIT.

\footnotetext{
${ }^{1}$ http://rise.hanyang.ac.kr
} 

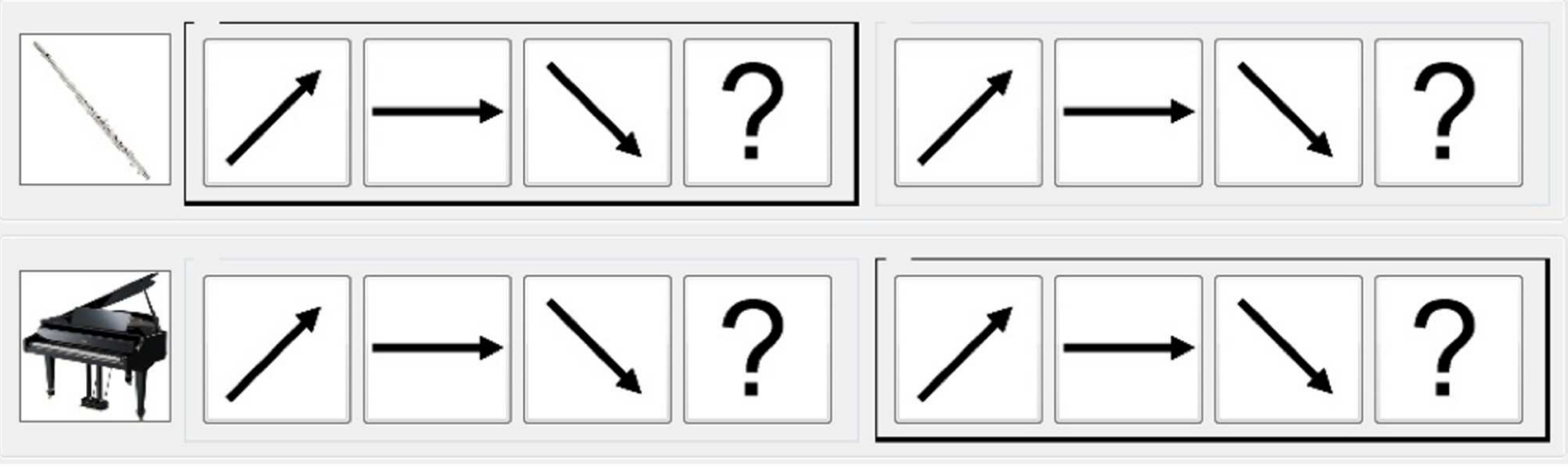

FIGURE 3 | Example of an answer sheet given in the CIT3.

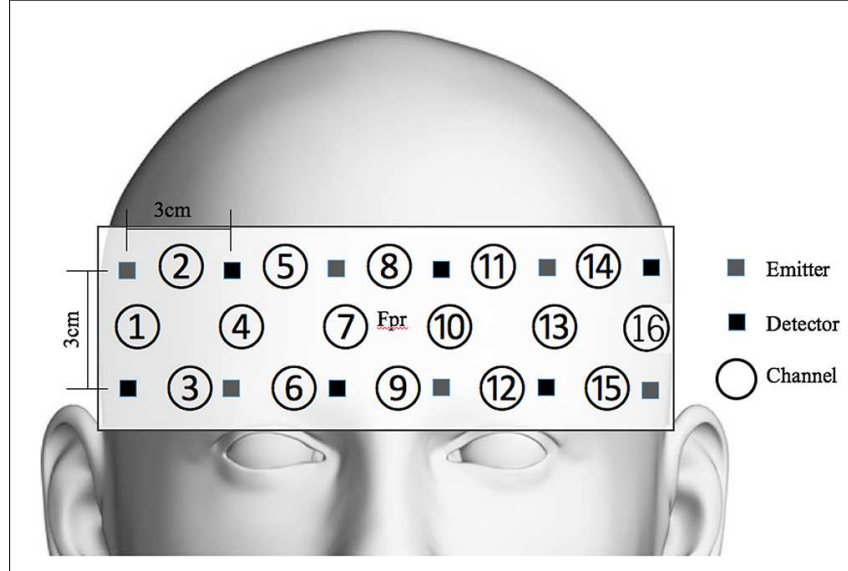

FIGURE 4 | Spectratech OEG-16. The center of the measurement unit was placed on the frontopolar region (Fpr) according to the international 10-20 system.
Each CIT began with approximately 15 seconds of instructions, followed by the 18 items. The given CIT task was to identify the directions of the target contour by clicking the arrows shown on the screen after the melodic contour was presented. All of the items were programmed using Visual Studio. The tasks were given in an order of task complexity (i.e., CIT1 to CIT3) and items were presented in a random order within each of the three CITs. The participants' behavioral and hemodynamic responses were recorded throughout the experiment. The CITs took about $20 \mathrm{~min}$ to complete. The experiment was performed in a sound-proof and light- and temperature-controlled room.

\section{Signal Processing and Statistical Analysis}

The fNIRS raw data were collected and converted into concentrated changes of hemoglobin using the modified BeerLambert law. Then, a zero-phase low- and high-pass filter with a cutoff frequency of 0.01 to $0.09 \mathrm{~Hz}$ was applied using MATLAB (Morren et al., 2004; Akgul et al., 2005; Bauernfeind et al., 2011).
Preprocessed oxyHb, which is known to be a sensitive indicator of cognitive load (Strangman et al., 2002; Holper and Wolf, 2011; Fishburn et al., 2014; Hwang et al., 2014; McKendrick et al., 2014), was averaged for pre-task, on-task, and post-task sessions. On-task sessions included three CITs, so the mean of oxyHb in each of the three CITs was estimated for statistical analyses.

A two-way mixed subject design was employed. The independent variables were age group (i.e., young vs. older), CIT, and session (pre-, on-, post tasks, for only NIRS data analysis). The dependent variables analyzed in this study were behavioral responses (performance accuracy, reaction time) and hemodynamic responses. We selected the following NIRS channels: channels 1 and 2 for right dorsolateral prefrontal cortex (DLPFC) activation and channels 14 and 15 for left DLPFC activation, which are known as sensitive regions to detect cognitive loads (Yasumura et al., 2014; Moriguchi et al., 2015). All statistical analyses were performed using Statistical Package for the Social Sciences (SPSS) ver. 20.

\section{RESULTS}

\section{Accuracy and Response Time}

Figure 5 shows the mean performance of the CIT with regard to accuracy (left panel, Figure 5A) and reaction time (right panel, Figure 5B) between the younger and older adult groups. The mean accuracy in the younger adult group was almost perfect for CIT1 given with the environmental sounds (99.1\%), followed by CIT2 given with a melodic distractor (93.2\%). Accuracy was the lowest $(89.7 \%)$ when a shift between two concurrent melodic contours was required (i.e., CIT3). A similar trend across the CITs was found for the older adult group (ranging from 32.1-67.1\%), but performance quickly decreased when performing both CIT2 and CIT3.

The percentage of correct responses was analyzed using group (young vs. older) x $\Delta$ CIT ( $\Delta$ CIT1-CIT2, $\Delta$ CIT2-CIT3) two-way mixed analysis of variance (ANOVA). $\triangle$ CITs were considered to represent extra cognitive loads corresponding to the changes 

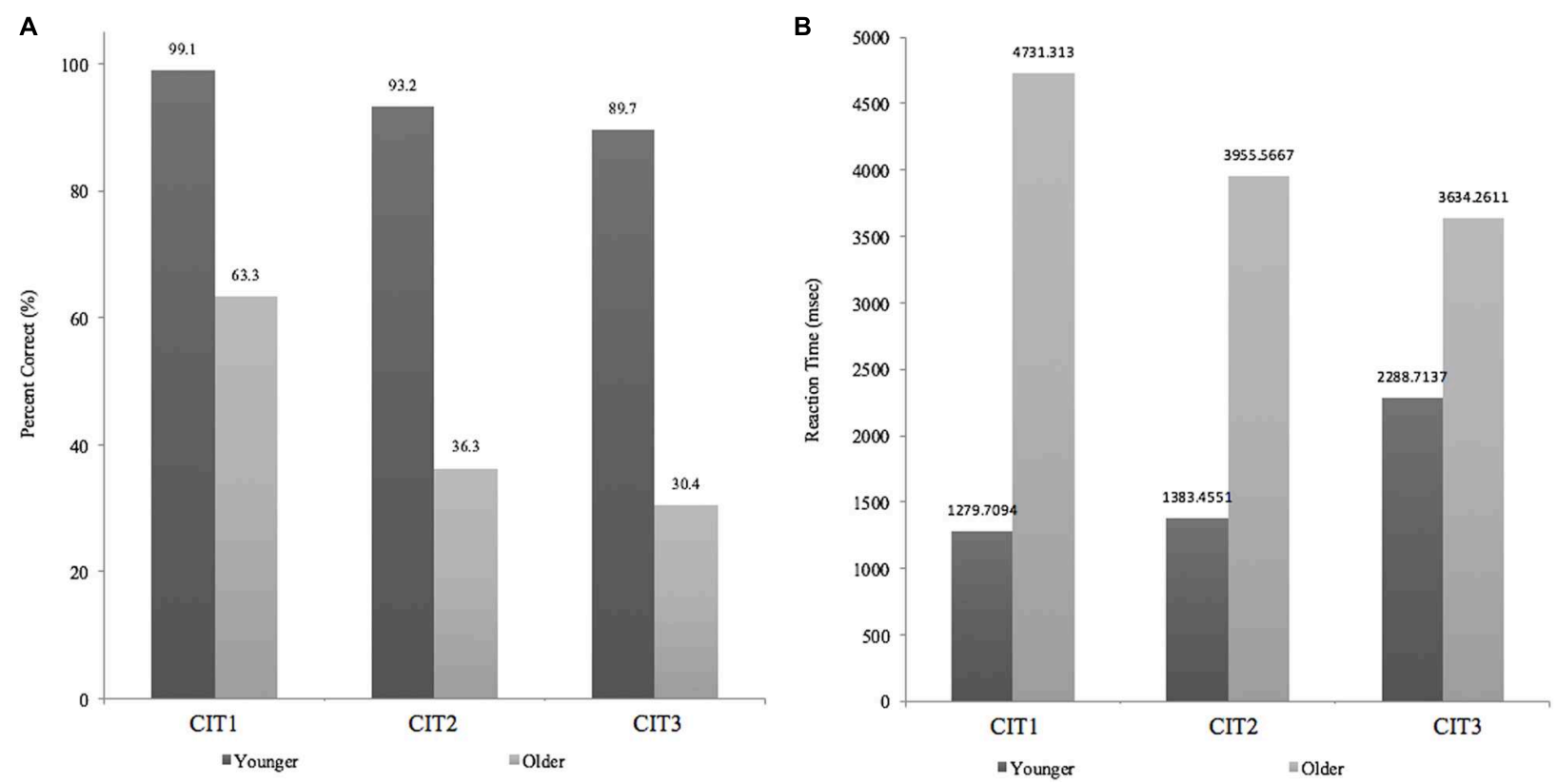

FIGURE 5 | (A) Accuracy changes across CITs (B) Reaction times across CITs.

in task difficulty among CITs, which might indicate how the two different age groups managed the three CITs. There was a significant main effect of group $\left(F_{1,25}=15.351, p<0.01\right)$ and $\Delta \operatorname{CIT}\left(F_{2,50}=14.273, p<0.01\right)$, indicating that the overall performance of the young adult group was better than that of the older adult group and also that $\Delta$ CIT1-CIT2 was greater than $\Delta$ CIT2-CIT3. In addition, we found a significant group $\times \Delta$ CIT two-way interaction $\left(F_{1,25}=9.262, p<0.01\right)$. Pairwise post hoc analysis of each $\Delta$ CIT (Bonferroni-Dunn's multiple comparison test) revealed a group difference at $\Delta$ CIT1-CIT2 $(p<0.001)$, indicating that the performance gap between CIT1 and CIT2, as compared to those between CIT2 and CIT3, was significantly higher for the older adult group $(p<0.001)$, while this was not the case for the younger adult group $(p>0.05, \mathrm{n} . \mathrm{s})$.

Figure 5B presents the reaction times between the two age groups, which showed opposite patterns but, either difference between groups or $\triangle$ CIT1-CIT2 and $\triangle$ CIT2-CIT3 were not significant. Reaction time was further analyzed using a group $\times \Delta$ CIT two-way mixed ANOVA. There was a significant main effect of group $\left(F_{1,25}=6.707, p<0.05\right)$ but no significant main effect of $\Delta$ CIT $\left(F_{1,25}=4.001, p>0.05\right)$ or an interaction effect $\left(F_{1,25}=1.375, p>0.05\right)$, indicating that older adults required more time to complete any identification task than did younger adults.

Taken together, these results show that both accuracy and reaction time suggest a clear distinction between the young and older adults. The significant interaction effect observed between $\triangle$ CIT1-CIT2 and group with regard to accuracy implies that CIT2 (i.e., melodic contour identification with a targetlike distraction) is sensitive enough to determine the difference between the young and the older age groups and those among three CITs in the older adult group.

\section{Hemodynamic Responses}

Hemodynamic responses were analyzed using oxyHb obtained from channels 1 and 2 for the right DLPFC and 14 and 15 for the left DLPFC. OxyHb was analyzed across pre-task (baseline), on-task (performing task), and post-task (baseline) sessions. The oxyHb at on-task was sectioned into three parts to examine the changes according to CIT. Table 2 presents the oxyHb changes across pre-, on-, and post-task sessions in the two different age groups. In general, oxyHb was increased compared to baseline after performing a task against baselines (i.e., pre-task and post-task), and this tendency differed by group.

The mean changes in oxyHb were analyzed using a group (young vs. older adults) $\times \Delta$ session ( $\Delta$ pre - on task, $\Delta$ on - post task) $\times$ channel $(\mathrm{CH} \mathrm{1,2,14,15)} \mathrm{mixed} \mathrm{ANOVA.} \mathrm{There} \mathrm{was} \mathrm{a}$ significant main effect of $\Delta$ session $\left(F_{1,25}=7.910, p<0.01\right)$, but the group and channel effects were not significant $\left(F_{1,25}=0.356\right.$, $p>0.05 ; F_{3,75}=1.773, p>0.05$, respectively). Interestingly, a significant interaction effect existed between group and $\Delta$ session $\left(F_{1,25}=4.401, p<0.05\right)$. A post hoc pairwise comparison revealed that both the $\Delta$ pre - on task and the $\Delta$ on - post task were significantly different only for the young adult group $(p<0.05, p<0.01$, respectively). These findings indicate that the sensitivity and flexibility of oxyHb concentration in response to a stimulus were greater in the young adult group than they were in the older adult group.

In the analysis of performance accuracy and response time, we found that CIT2 was of vital importance for determining the difference between the young and the older age groups and can be used as a critical task to represent cognitive functioning in the elderly. Therefore, we again compared the changes in oxyHb concentration between pre-task and CIT2 ( $\Delta$ Pre- CIT2) in order 
TABLE 2 | Descriptive statistics of oxyHb concentration.

\begin{tabular}{|c|c|c|c|c|c|c|}
\hline \multirow[t]{3}{*}{ Session } & \multirow[t]{3}{*}{ CIT } & \multirow[t]{3}{*}{ Channel } & \multicolumn{4}{|c|}{ Group } \\
\hline & & & \multicolumn{2}{|c|}{ Younger } & \multicolumn{2}{|c|}{ Older } \\
\hline & & & $M$ & $S D$ & $M$ & $S D$ \\
\hline \multirow[t]{5}{*}{ Pre-task } & & 1 & -0.0383 & 0.0440 & -0.0102 & 0.0095 \\
\hline & & 2 & -0.0298 & 0.0546 & -0.0070 & 0.0515 \\
\hline & & 14 & -0.0241 & 0.0378 & -0.0117 & 0.0423 \\
\hline & & 15 & -0.0260 & 0.0712 & 0.0069 & 0.0642 \\
\hline & & Average & -0.02955 & 0.0519 & -0.0055 & 0.0419 \\
\hline \multirow[t]{15}{*}{ On-task } & ClT1 & 1 & 0.0037 & 0.0088 & 0.0007 & 0.0041 \\
\hline & & 2 & 0.0031 & 0.0039 & -0.0002 & 0.0029 \\
\hline & & 14 & 0.0015 & 0.0054 & 0.0001 & 0.0021 \\
\hline & & 15 & 0.0007 & 0.0054 & -0.0005 & 0.0037 \\
\hline & & Average & 0.0023 & 0.0059 & 0.0000 & 0.0032 \\
\hline & CIT2 & 1 & 0.0007 & 0.0058 & 0.0002 & 0.0017 \\
\hline & & 2 & -0.0009 & 0.0048 & -0.0000 & 0.0020 \\
\hline & & 14 & -0.0026 & 0.0047 & 0.0002 & 0.0032 \\
\hline & & 15 & -0.0031 & 0.0058 & 0.0006 & 0.0042 \\
\hline & & Average & -0.0015 & 0.0053 & 0.0003 & 0.0028 \\
\hline & CIT3 & 1 & 0.0037 & 0.0088 & 0.0008 & 0.0032 \\
\hline & & 2 & 0.0031 & 0.0039 & 0.0000 & 0.0027 \\
\hline & & 14 & 0.0015 & 0.0054 & -0.0006 & 0.0021 \\
\hline & & 15 & 0.0007 & 0.0054 & 0.0010 & 0.0031 \\
\hline & & Average & 0.0023 & 0.0059 & 0.0003 & 0.0028 \\
\hline \multirow[t]{5}{*}{ Post-task } & & 1 & -0.0472 & 0.1171 & -0.0035 & 0.0292 \\
\hline & & 2 & 0.0203 & 0.0602 & 0.0053 & 0.0513 \\
\hline & & 14 & 0.0138 & 0.0556 & 0.0046 & 0.0410 \\
\hline & & 15 & -0.0308 & 0.1210 & -0.0064 & 0.0293 \\
\hline & & Average & -0.0110 & 0.0885 & 0 & 0.0377 \\
\hline
\end{tabular}

to determine if fNIRS data were consistent with the difference in behavioral performance (Figure 6).

The mean changes in $\triangle \mathrm{Pre}-\mathrm{CIT} 2 \mathrm{oxyHb}$ were analyzed using a group $\times$ channel $(1,2,14,15)$ mixed ANOVA. There was a significant main effect of group $\left(F_{1,25}=4.882, p<0.05\right)$. Further, we performed separate independent sample $t$-tests at each channel. $\Delta$ Pre-CIT2 oxyHb on Channel 1 was greater in the young adult group than in the older adult group $[t(25)=-2.336$, $p<0.05]$. These results suggest that the group difference at $\Delta$ PreCIT2 was mostly due to the oxyHb changes that were increased in the right DLPFC in the younger adult group.

The changes in oxyHb concentration indicated that the young adults were more sensitive (as shown in $\Delta$ Pre-on task and $\Delta$ PreCIT2) and flexible ( $\Delta$ On-Post task) at performing melodic CITs. This tendency was in agreement with the participants' behavioral performances (i.e., their accuracy and response times). In effect, CIT2 is a promising task to determine the difference between younger and older age groups and that within an older adults group (e.g., some elderly people may cope well with CIT2). The right DLPFC was a prominent brain area for detecting such a tendency due to the main characteristics of the given tasks. (Note that CITs are melodic CITs in which spatial cognition is vital. Also, the DLPFC is involved in complex cognitive functions such as executive functioning.)

\section{DISCUSSION}

The present study provides behavioral and neurophysiological evidence of the differences in melodic contour identification performance between younger and older adults. The behavioral results revealed that the cognitive declines observed in the older age group were obvious when participants were faced with a target surrounded by competing distraction (i.e., CIT2). Consistently, our fNIRS data showed a higher level of activation to the CITs in the younger age group than in older adults. The oxyHb concentration changes between the two groups were also greater when processing CIT2 at the right DLPFC (Channel 1).

\section{Behavioral Results}

Overall, CITs using melodic contours in this study were shown to adequately indicate age-related cognitive declines. In particular, CIT2 (i.e., a selective listening task to target contours against target-like distractors) was considered a potential testing task to indicate a certain type of cognitive decline that typically occurs with aging. In general, accuracy gradually decreased across CITs and was lower in the older than in the younger adults. This finding is similar to those of previous studies that have reported that melodic contour identification performance decreased with aging (Mukari et al., 2010). This tendency is also consistent with previous music perception studies that have reported that such changes in music are probably due to decreased capacity in temporal and spatial resolution (Tremblay et al., 2003; ParberyClark et al., 2011). Neurological evidence has further indicated that the changes in connectivity and thickness of the insular cortex with aging (Heuninckx et al., 2005; Wong et al., 2009; Churchwell and Yurgelyn-Todd, 2013) might lead to decreased CIT performance.

One remarkable finding in this study is that a group difference was noted between CIT1 and CIT2, rather than CIT2 and CIT3. It is generally assumed that mental flexibility or shifting is more difficult than selective attention. However, changes between these two cognitive functions were not obvious in the older adult group, indicating when selective attention is intact or less affected, alternating attention is used for an indicator of cognitive evaluation. When it is affected, evaluation of the more complex cognitive function is meaningless -since the functions are posited in a hierarchy and if a fundamental function is not working, a higher one consequently cannot work.

Further, this tendency was more prominent in the older than in the younger adult group. These findings were possibly due to a reduced ability for timbre discrimination. Timbre is one of the key factors of ASA, as it provides a perceptual clue to how one organizes perceived auditory surroundings (Bregman, 1999; Neuhoff, 2011). For example, when two 


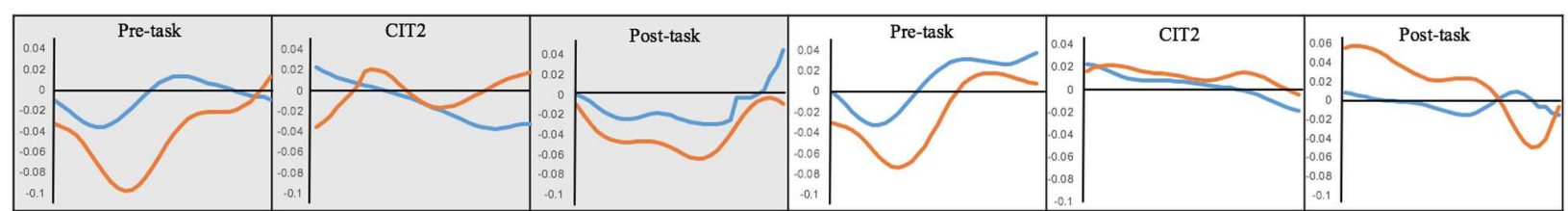

Channel 1

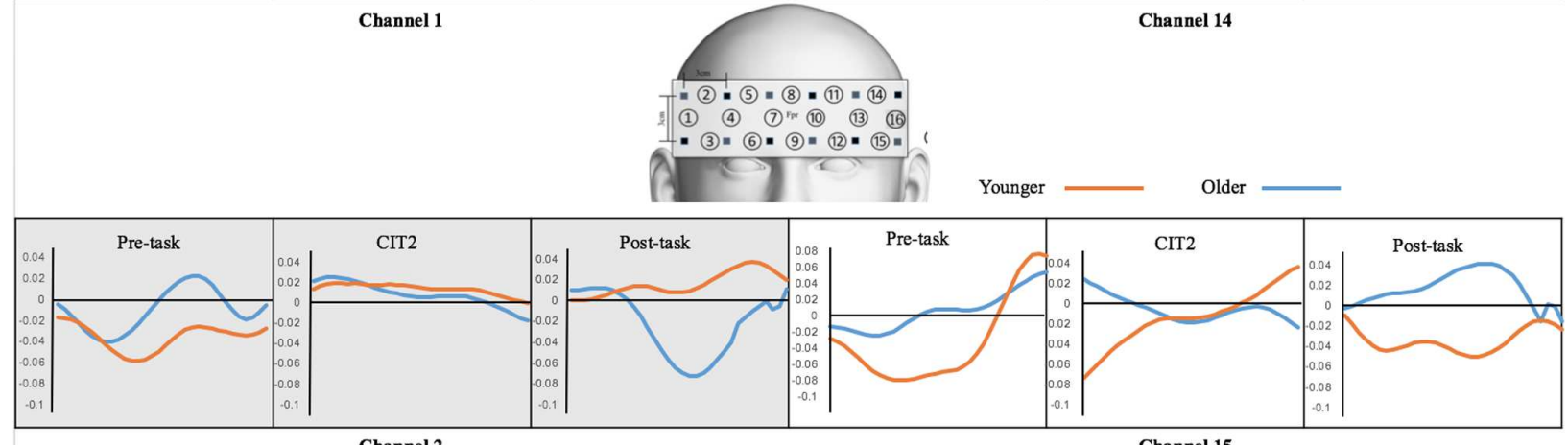

Channel 14

Channel 2

Channel 15

FIGURE 6 | Changes in oxyHb concentration. OxyHb obtained from the pre-task, CIT2, and post-task were compared between the younger and older adult groups.

people speak simultaneously, we can typically distinguish between them based on voice timbre. Such timbre cues along with other pitch and loudness information, aid parsing, and tracking allow for distinction of the target voice in a complex auditory environment. This interpretation is very similar to those of previous studies that reported that listening to multilayered sounds, as an example of a cocktail party effect, is quite challenging with aging (Heine and Browning, 2002; Parbery-Clark et al., 2009b, 2011). A person's ASA capacity rapidly decreases with aging, leading to loss of speech comprehension (Grassi and Borella, 2013; Arehart et al., 2014).

Higher-order cognitive processes, such as attention, stored knowledge, and goals, operate in ASA in concert with timbre discrimination (Alain et al., 2001). As described in Russo and Pichora-Fuller (2008), younger adults were more flexible in their ability to simultaneously zoom in and out to attend target and non-target sounds. Speech studies in the noise perception literature are also in line with the music studies mentioned above, indicating that the poorer performance of older adults has been attributed to declines in both hearing sensitivity (Russo and Pichora-Fuller, 2008; Parbery-Clark et al., 2011; Vermeire et al., 2015) and cognitive function (Guerreiro et al., 2010).

\section{fNIRS Results}

Our findings were novel in that oxyHb activation showed musicspecific PFC activation. Across sessions (pre-task, CITs, posttask), the oxyHb concentration summed from all four channels increased with CIT performance and returned to baseline at CIT completion. The discrepancies between baseline and CITs and between CITs back to baseline were much greater with younger than older adults. Also, as expected, the facilitating effect of music on CIT performance resulted in a higher oxyHb concentration in the right DLPFC. In other words, the oxyHb concentration in the right DLPFC greatly increased during CIT performance (i.e., $\triangle$ Pre- CIT2), specifically for the younger adults. The current findings are indicative of changes in degree and type of cognitive capacity with aging.

The oxyHb increases in the right DLPFC between Pre-task and CIT2 are reflective of the essential characteristics of CITs. That is, the CITs in this study consisted of melodic contours, so the participants listened repeatedly to short melodies. The higher level of activation that was observed in the right hemisphere $(\mathrm{RH})$ is in line with previous neuroimaging studies in which pitch pattern perception activated cognitive resources in the $\mathrm{RH}$ in an automatic and controlled manner (Harrington, 1987; Trainor et al., 2002; Zatorre et al., 2002; Lee et al., 2011). The PFC lateralization specific to task types (verbal recognition, Cabeza et al., 2003) and stuttering types (Kazenski, 2015) has been studied using fNIRS, but no investigations have focused on music processing. Hence, our findings firstly suggest right lateralization of the PFC in processing melodic contours provided in the selective listening task. More specifically, the higher level of right DLPFC enhancement between pre-task and CIT2 that was observed only in younger adults was in agreement with previous fNIRS studies that have revealed that older adults show less hemispheric lateralization in given cognitive tasks compared to younger adults (Tsjuii et al., 2010).

Second, our fNIRS data indicate that oxyHb concentration was more sensitive, ready for activation, and flexible to return to the neutral state in the younger than older adults. This finding is in agreement with previous fNIRS studies that claimed that older adults achieve a lower plateau of oxyHb regardless of any increase in task complexity, while younger adults tend to flexibly cope with task complexity increases (Mattay et al., 2006; Missonnier et al., 2011; Nagel et al., 2011). Other fNIRS studies have also supported our findings that younger adults showed higher activation in the PFC oxyHb concentration when performing more complex 
cognitive tasks compared to older adults (Herrmann et al., 2006; Vermeij et al., 2012).

These results can be further interpreted with regard to the roles of DLPFC. It is known that the DLPFC is activated by additional cognitive load so it can effectively modulate executive control and working memory (Opitz et al., 2002, 2005; Gaab et al., 2003; Barbey et al., 2013). In auditory modalities, the DLPFC is also consistently activated as the cognitive load increases (Alain et al., 2001; Seydell-Greenwald et al., 2013; Morita et al., 2015). In this sense, Lipschutz et al. (2002) claimed that greater DLPFC activation would be expected when performing a more complex cognitive task. Therefore, the changes in oxyHb concentrations that were noted in the right DLPFC (i.e., Channel 1) reflect both stimuli and task characteristics of the CITs.

\section{Conclusion and Future Suggestions}

In conclusion, our findings showed that CIT performance could reflect the cognitive differences that exist between age groups. Note that younger adults performed well in the shifting tasks (i.e., CIT3); however, older adults showed a significant decrease in the selective attention task performed in conjunction with competing distractors (i.e., CIT2). Therefore, our findings imply that the detrimental cognitive functioning that occurs with aging can be effectively detected by the selective attention task rather than by shifting attention (i.e., flexibility) in music CITs. Also, our fNIRS data suggest that this tendency can be easily detected in the right DLPFC, indicating that declines in selective auditory attention and speech-in-noise perception are possibly associated with this region.

We recognize some limitations of the present study. The fNIRS was devised to observe PFC activation alone, so any activations in other brain regions and also the relationships between these regions and the PFC have not been accounted for; we therefore cannot determine whether cognitive declines that are associated with aging are solely dependent on the role of the

\section{REFERENCES}

Akgul, C. B., Sankur, B., and Akin, A. (2005). Spectral analysis of event-related hemodynamic responses in functional near infrared spectroscopy. J. Comput. Neurosci. 18, 67-83. doi: 10.1007/s10827-005-5478-2

Alain, C., Arnott, S. R., and Picton, T. W. (2001). Bottom-up and topdown influences on auditory scene analysis: evidence from event-related brain potentials. J. Exp. Psychol. Hum. Percept. Perform. 27, 1072-1089. doi: 10.1037/0096-1523.27.5.1072

Alain, C., Quan, J., McDonald, K., and van Roon, P. (2009). Noise-induced increase in human auditory evoked neuromagnetic fields. Eur. J. Neurosci. 30, 132-142. doi: 10.1111/j.1460-9568.2009.06792.x

Allen, K., Alais, D., Shinn-Cunningham, B., and Carlile, S. (2011). Masker location uncertainty reveals evidence for suppression of maskers in twotalker contexts. J. Acoust. Soc. Am. 130, 2043-2053. doi: 10.1121/1.363 1666

Arehart, K. H., Croghan, N. B., and Muralimanohar, R. K. (2014). Effects of age on melody and timbre perception in simulations of electro-acoustic and cochlearimplant hearing. Ear. Hear. 35, 195-202. doi: 10.1097/AUD.0b013e3182a69a5c

Barbey, A. K., Koenigs, M., and Grafman, J. (2013). Dorsolateral prefrontal cortex contributions to human working memory. Cortex 49, 1195-1205. doi: 10.1016/j.cortex.2012.05.022
PFC. Instead, this study focused on a non-verbal standardized evaluation task (note that musical non-verbal information processing is expected to be less knowledge- and culturedependent), which empirically tests the validity of the CITs. An urgent future study would thus be a scaled-up experiment to examine the relationship between music and cognitive function in real-world situations. Another type of future study might be the use of CITs in patients with cognitive impairment (e.g., dementia, mild cognitive impairment). The end goal is to develop an early screening test for age-related cognitive functioning after determining how melodic contour can be efficiently employed in the clinical setting.

\section{AUTHOR CONTRIBUTIONS}

HR: Substantial contributions to the conception of the work; Revising it critically for important intellectual content; The interpretation of data for the work; Final approval of the version to be published; Agreement to be accountable for all aspects of the work in ensuring that questions related to the accuracy or integrity of any part of the work are appropriately investigated and resolved. EJ: Substantial contributions to the design of the work; The acquisition, analysis, and interpretation of data for the work; Drafting and revising the manuscript; Final approval of the version to be published; Agreement to be accountable for all aspects of the work in ensuring that questions related to the accuracy or integrity of any part of the work are appropriately investigated and resolved.

\section{FUNDING}

This work was supported by the National Research Foundation of Korea Grant funded by the Korean Government (NRF2014R1A2A2A01002583).

Bauernfeind, G., Scherer, R., Pfurtscheller, G., and Neuper, C. (2011). Singletrial classification of antagonistic oxyhemoglobin responses during mental arithmetic. Med. Biol. Eng. Comput. 49, 979-984. doi: 10.1007/s11517-0110792-5

Belleville, S., Menard, M. C., and Lepage, E. (2011). Impact of novelty and type of material on recognition in healthy older adults and persons with mild cognitive impairment. Neuropsychologia 49, 2856-2865. doi: 10.1016/j.neuropsychologia.2011.06.011

Best, V., Carlile, S., Kopco, N., and van Schaik, A. (2011). Localization in speech mixtures by listeners with hearing loss. J. Acoust. Soc. Am. 129, 210-215. doi: $10.1121 / 1.3571534$

Bones, O., Hopkins, K., Krishnan, A., and Plack, C. J. (2014). Phase locked neural activity in the human brainstem predicts preference for musical consonance. Neuropsychologia $\quad 58, \quad 23-32$. doi: 10.1016/j.neuropsychologia.2014. 03.011

Bones, O., and Plack, C. J. (2015). Losing the music: aging affects the perception and subcortical neural representation of musical harmony. J. Neurosci. 35, 4071-4080. doi: 10.1523/JNEUROSCI.3214-14.2015

Bregman, A. S. (1999). "Auditory organization in music", in Auditory Scene Analysis: The Perceptual Organization of Sound, ed. A. S. Bregman (Cambridge, MA: MIT Press), 455-528.

Broadbent, D. (1958). Perception and Communication. London: Pergamon Press. 
Cabeza, R., Locantore, J. K., and Anderson, N. D. (2003). Lateralization of prefrontal activity during episodic memory retrieval: evidence for the production-monitoring hypothesis. J. Cogn. Neurosci. 15, 249-259. doi: 10.1162/089892903321208187

Churchwell, J. C., and Yurgelyn-Todd, D. A. (2013). Age-related changes in insula cortical thickness and impulsivity: significance for emotional development and decision-making. Dev. Cogn. Neurosci. 6, 80-86. doi: 10.1016/j.dcn.2013.07.001

Cuddy, L. L., and Duffin, J. (2005). Music, memory and Alzheimer's disease: is music recognition spared in dementia, and how can it be assessed? Med. Hypotheses 64, 229-235. doi: 10.1016/j.mehy.2004.09.005

De Oliveira, T. C., Soares, F. C., De Macedo, L. D., Picanço-Diniz, D. L., Bento-Torres, N. O., and Picanço-Diniz, C. W. (2014). Beneficial effects of multisensory and cognitive stimulation on age-related cognitive decline in longterm-care institutions. Clin. Interv. Aging 9, 309-321. doi: 10.2147/CIA.S54383

Deary, I. J., Corley, J., Gow, A. J., Harris, S. E., Houlihan, L. M., Marioni, R. E., et al. (2009). Age-associated cognitive decline. Br. Med. Bull. 92, 135-152. doi: $10.1093 / \mathrm{bmb} / \mathrm{ldp} 033$

Faubert, J. (2002). Visual perception and aging. Can. J. Psychol. 56, 164-176. doi: $10.1037 / \mathrm{h} 0087394$

Fedorenko, E., Patel, A., Casasanto, D., Winawer, J., and Gibson, E. (2009). Structural integration in language and music: evidence for a shared system. Mem. Cogn. 37, 1-9. doi: 10.3758/MC.37.1.1

Ferrari, M., and Quaresima, V. (2012). A brief review on the history of human functional near-infrared spectroscopy (fNIRS) development and fields of application. Neuroimage 63, 921-935. doi: 10.1016/j.neuroimage.2012.03.049

Fishburn, F. A., Norr, M. E., Medvedev, A. V., and Vaidya, C. J. (2014). Sensitivity of fNIRS to cognitive state and load. Front. Hum. Neurosci. 8:76. doi: 10.3389/fnhum.2014.00076

Francis, A. L. (2010). Improved segregation of simultaneous talkers differentially affects perceptual and cognitive capacity demands for recognizing speech in competing speech. Atten. Percept. Psychophys. 72, 501-516. doi: 10.3758/APP.72.2.501

Gaab, N., Gaser, C., Zaehle, T., Jancke, L., and Schlaug, G. (2003). Functional anatomy of pitch memory: an fMRI study with sparse temporal sampling. Neuroimage 19, 1417-1426. doi: 10.1016/S1053-8119(03)00224-6

Galvin, J. J., Fu, Q., and Oba, S. (2008). Effect of instrument timbre on melodic contour identification by cochlear implant user. J. Acoust. Soc. Am. 124, 189195. doi: 10.1121/1.2961171

Galvin, J. J., Fu, Q., and Oba, S. I. (2009). Effect of a competing instrument on melodic contour identification by cochlear implant user. J. Acoust. Soc. Am. 125, 98-103. doi: 10.1121/1.3062148

Gilbert, S. J., Frith, C. D., and Burgess, P. W. (2005). Involvement of rostral prefrontal cortex in selection between stimulus-oriented and stimulusindependent thought. Eur. J. Neurosci. 21, 1423-1431. doi: 10.1111/j.14609568.2005.03981.x

Grassi, M., and Borella, E. (2013). The role of auditory abilities in basic mechanisms of cognition in older adults. Front. Aging Neurosci. 5:59. doi: 10.3389/fnagi.2013.00059

Guerreiro, M., Murphy, D., and Van Gerven, P. (2010). Role of sensory modality in age-related distraction: a critical review and a renewed view. Psychol. Bull. 136, 975-1022. doi: 10.1037/a0020731

Harrington, A. (1987). Medicine, Mind, and the Double Brain: A Study in NinteenthCentury Thought. Princeton, NJ: Princeton University Press.

Heine, C., and Browning, C. (2002). Communication and psychosocial consequences of sensory loss in older adults: overview and rehabilitation directions. Disabil. Rehabil. 24, 763-773. doi: 10.1080/09638280210129162

Heinrich, A., Schneider, B. A., and Craik, F. I. (2008). Investigating the influence of continuous babble on auditory short-term memory performance. Q. J. Exp. Psychol. 61, 735-751. doi: 10.1080/17470210701402372

Herrmann, M. J., Walter, A., Ehlis, A. C., and Fallgatter, A. J. (2006). Cerebral oxygenation changes in the prefrontal cortex: effects of age and gender. Neurobiol. Aging 27, 888-894. doi: 10.1016/j.neurobiolaging.2005.04.013

Heuninckx, S., Wenderoth, N., Debaere, F., Peeters, R., and Swinnen, S. P. (2005). Neural basis of aging: the penetration of cognition into action control. J. Neurosci. 25, 6787-6796. doi: 10.1523/JNEUROSCI.1263-05.2005

Holper, L., and Wolf, M. (2011). Single-trial classification of motor imagery differing in task complexity: a functional near-infrared spectroscopy study. J. Neuroeng. Rehabil. 8:34. doi: 10.1186/1743-0003-8-34
Hwang, H. J., Lim, J. H., Kim, D. W., and Im, C. H. (2014). Evaluation of various mental task combinations for near-infrared spectroscopybased brain-computer interfaces. J. Biomed. Optics. 19, 077005. doi: 10.1117/1.JBO.19.7.077005

Inspector, M., Manor, D., Amir, N., Kushnir, T., and Karni, A. (2013). A word by any other intonation: fMRI evidence for implicit memory traces for pitch contours of spoken words in adult brains. PLOS ONE 8:e82042. doi: 10.1371/journal.pone.0082042

Janata, P. (2005). Brain networks that track musical structure. Ann. N. Y. Acad. Sci. 1060, 111-124. doi: 10.1196/annals.1360.008

Janata, P., Tillmann, B., and Bharucha, J. J. (2002). Listening to polyphonic music recruits domain-general attention and working memory circuits. Cogn. Affect. Behav. Neurosci. 2, 121-140. doi: 10.3758/CABN.2.2.121

Jeong, E. (2013). Psychometric validation of a music-based attention assessment: revised for patients with traumatic brain injury. J. Music. Ther. 50, 66-92. doi: $10.1093 / \mathrm{jmt} / 50.2 .66$

Jeong, E., and Lesiuk, T. (2011). Development and preliminary evaluation of a music-based attention assessment for patients with traumatic brain injury. J. Music. Ther. 48, 255-271. doi: 10.1093/jmt/48.4.551

Jeong, E., and Ryu, H. (2016). Nonverbal auditory working memory: does music can indicated the capacity? Brain. Cogn. 105, 9-21. doi: 10.1016/j.bandc.2016.03.003

Jobsis, F. F. (1977). Noninvasive, infrared monitoring of cerebral and myocardial oxygen sufficiency and circulatory parameters. Science 198, 1264-1267. doi: $10.1126 /$ science.929199

Kazenski, D. M. (2015). fNIRS Measures of Prefrontal Cortex Lateralization during Stuttered and Fluency-Enhanced Speech in Adults Who Stutter. Ph.D. dissertation, University of Vermont, Burlington VT.

Kelley, W. M., Ojemann, J. G., Wetzel, R. D., Derdeyn, C. P., Moran, C. J., Cross, D. T., et al. (2002). Wada testing reveals frontal lateralization for the memorization of words and faces. J. Cogn. Neurosci. 14, 116-125. doi: $10.1162 / 089892902317205375$

Lee, Y. S., Janata, P., Frost, C., Hanke, M., and Granger, R. (2011). Investigation of melodic contour processing in the brain using multivariate pattern-based fMRI. Neuroimage 57, 293-300. doi: 10.1016/j.neuroimage.2011. 02.006

Leitman, D. I., Sehatpour, P., Shpaner, M., Foxe, J. J., and Javitt, D. C. (2009). Mismatch negativity to tonal contours suggests preattentive perception of prosodic content. Brain. Imaging Behav. 3, 284-291. doi: 10.1007/s11682-0099070-7

Lipschutz, B., Kolinsky, R., Damhaut, P., Wikler, D., and Goldman, S. (2002). Attention-dependent changes of activation and connectivity in dichotic listening. Neuroimage 17, 643-656. doi: 10.1006/nimg.2002.1184

Loui, P., Guenther, F. H., Mathys, C., and Schlaug, G. (2008). Actionperception mismatch in tone-deafness. Curr. Biol. 18, 331-332. doi: 10.1016/j.cub.2008.02.045

Luo, X., Masterson, M. E., and Wu, C. C. (2014a). Contour identification with pitch and loudness cues using cochlear implants. J. Acoust. Soc. Am. 135, 8-14. doi: $10.1121 / 1.4832915$

Luo, X., Masterson, M. E., and Wu, C. C. (2014b). Melodic interval perception by normal hearing listeners and cochlear implant users. J. Acoust. Soc. Am. 136, 1831-1844. doi: 10.1121/1.4894738

Mattay, V. S., Fera, F., Tessitore, A., Hariri, A. R., Berman, K. F., Das, S., et al. (2006). Neurophysiological correlates of age-related changes in working memory capacity. Neurosci. Lett 392, 32-37. doi: 10.1016/j.neulet.2005. 09.025

McKendrick, R., Ayaz, H., Olmstead, R., and Parasuraman, R. (2014). Enhancing dual-task performance with verbal and spatial working memory training: continuous monitoring of cerebral hemodynamics with NIRS. Neuroimage 85(Pt3), 1014-1026. doi: 10.1016/j.neuroimage.2013. 05.103

Missonnier, P., Herrmann, F. R., Rodriguez, C., Deiber, M. P., Millet, P., Faziocosta, L., et al. (2011). Age-related differences on event-related potentials and brain rhythm oscillations during working memory activation. J. Neural. Transm. 118, 945-955. doi: 10.1007/s00702-011-0600-2

Moriguchi, Y., Sakata, Y., Ishibashi, M., and Ishikawa, Y. (2015). Teaching others rule-use improved executive function and prefrontal activations in young children. Front. Psychol. 6:894. doi: 10.3389/fpsyg.2015.00894 
Morita, A., Morishima, Y., and Rackham, D. W. (2015). Right dorsolateral prefrontal cortex activation during a time production task: a functional nearinfrared spectroscopy study. Asian J. Neurosci. 2015:189060. doi: 10.1007/9781-4614-7411-1_11

Morren, G., Wolf, M., Lemmerling, P., Wolf, U., Choi, J. H., Gratton, E., et al. (2004). Detection of fast neuronal signals in the motor cortex from functional near infrared spectroscopy measurements using independent component analysis. Med. Biol. Eng. Comput. 42, 92-99. doi: 10.1007/BF02351016

Mukari, S. Z., Umat, C., and Othman, N. J. (2010). Effects of age and working memory capacity on pitch pattern sequence test and dichotic listening. Audiol. Neurotol. 15, 303-310. doi: 10.1159/000283007

Nagel, I. E., Preuschhof, C., Li, S. C., Nyberg, L., Bäckman, L., Lindenberger, U., et al. (2011). Load modulation of the BOLD response and connectivity predicts working memory performance in younger and older adults. J. Cogn. Neurosci. 23, 2030-2045. doi: 10.1162/jocn.2010.21560

Neuhoff, C. (2011). "Perception, cognition and action in auditory displays," in The Sonification Handbook, eds T. Hermann, A. Hunt, and C. Neuhoff (Berlin: Logos Publishing House), 63-85.

Ochsner, K. N., and Gross, J. J. (2005). The cognitive control of emotion. Trends Cog. Sci. 9, 242-249. doi: 10.1016/j.tics.2005.03.010

Ogawa, Y., Kotani, K., and Jimbo, Y. (2014). Relationship between working memory performance and neural activation measured using near-infrared spectroscopy. Brain Behav. 4, 544-551. doi: 10.1002/brb3.238

Oldfield, R. C. (1971). The assessment and analysis of handedness: the Edinburgh inventory. Neuropsychologia 9, 97-113. doi: 10.1016/0028-3932(71)90067-4

Opitz, B., Rinne, T., Mecklinger, A., von Cramon, D. Y., and Schröger, E. (2002). Differential contribution of frontal and temporal cortices to auditory change detection: fMRI and ERP results. Neuroimage 15, 167-174. doi: 10.1006/nimg.2001.0970

Opitz, B., Schröger, E., and von Cramon, Y. (2005). Sensory and cognitive mechanisms for pre-attentive change detection in auditory cortex. Eur. J. Neurosci. 21, 531-535. doi: 10.1111/j.1460-9568.2005.03839.x

Ostroff, J. M., McDonald, K. L., Schneider, B. A., and Alain, C. (2003). Aging and the processing of sound duration in human auditory cortex. Hear. Res. 181, 1-7. doi: 10.1016/S0378-5955(03)00113-8

Parbery-Clark, A., Skoe, E., and Kraus, N. (2009a). Musical experience limits the degradative effects of background noise on the neural processing of sound. J. Neurosci. 29, 14100-14107. doi: 10.1523/JNEUROSCI.3256-09.2009

Parbery-Clark, A., Skoe, E., Lam, C., and Kraus, N. (2009b). Musician enhancement for speech-in-noise. Ear. Hear. 30, 653-661. doi: 10.1097/AUD.0b013e3181b412e9

Parbery-Clark, A., Strait, D. L., Anderson, S., Hittner, E., and Kraus, N. (2011). Musical experience and the aging auditory system: implications for cognitive abilities and hearing speech in noise. PLOS ONE 6:e18082. doi: 10.1371/journal.pone.0018082

Patel, A. D. (2008). Music, Language, and the Brain. New York, NY: Oxford University Press.

Peck, E. M., Yuksel, B. F., Ottley, A., Jacob, R. J., and Chang, R. (2013). "Using fNIRS brain sensing to evaluate information visualization interfaces," in Proceedings of the 2013 Annual Conference on Human Factors in Computing Systems, New York, NY.

Pichora-Fuller, M. K. (2003). Cognitive aging and auditory information processing. Int. J. Audiol. 42(Suppl.2), 2S26-2S32. doi: 10.3109/14992020309074641

Pichora-Fuller, M. K. (2006). "Audition and cognition: what audiologists need to know about listening," in Hearing Care for Adults, eds C. Palmer and R. Seewald (Stäfa: Phonak), 71-85.

Platel, H., Baron, J. C., Desgranges, B., Bernard, F., and Eustache, F. (2003). Semantic and episodic memory of music are subserved by distinct neural networks. Neuroimage 20, 244-256. doi: 10.1016/S1053-8119(03)00287-8

Rahman, T. T., Mohamed, S. T., Albanouby, M. H., and Bekhet, H. F. (2011). Central auditory processing in elderly with mild cognitive impairment. Geriatr. Gerontol. 11, 304-308. doi: 10.1111/j.1447-0594.2010.00684.x

Russo, F., and Pichora-Fuller, M. K. (2008). Tune in or tune out: age-related differences in listening to speech in music. Ear. Hear. 29, 746-760. doi: 10.1097/AUD.0b013e31817bdd1f

Sato, H., Yahata, N., Funane, T., Takizawa, R., Katura, T., Atsumori, H., et al. (2013). A NIRS-fMRI investigation of prefrontal cortex activity during a working memory task. Neuroimage $83,158-173$.
Satoh, M., Takeda, K., Nagata, K., Hatazawa, J., and Kuzuhara, S. (2001). Activated brain regions in musicians during an ensemble: a PET study. Cogn. Brain Res. 12, 101-108. doi: 10.1016/S0926-6410(01)00044-1

Schirmer, A., and Kotz, S. A. (2006). Beyond the right hemisphere: brain mechanisms mediating vocal emotional processing. Trends Cogn. Sci. 10, 24-30. doi: 10.1016/j.tics.2005.11.009

Seydell-Greenwald, A., Greenberg, A. S., and Rauschecker, J. P. (2013). Are you listening? Brain activation associated with sustained nonspatial auditory attention in the presence and absence of stimulation. Hum. Brain. Mapp. 35 2233-2252. doi: 10.1002/hbm.22323

Shinn-Cunningham, B. G., and Best, V. (2008). Selective attention in normal and impaired hearing. Trends Amplif. 12, 283-299. doi: 10.1177/1084713808325306

Snyder, J. S., and Alain, C. (2005). Age-related changes in neural activity associated with concurrent vowel segregation. Cogn. Brain Res. 24, 492-499. doi: 10.1016/j.cogbrainres.2005.03.002

Strangman, G., Culver, J. P., Thompson, J. H., and Boas, D. A. (2002). Quantitative comparison of simultaneous BOLD fMRI and NIRS recordings during functional brain activation. Neuroimage 17, 719-731. doi: 10.1006/nimg.2002.1227

Thompson, W. F., and Schellenberg, G. (2006). "Listening to Music," in MENC Handbook of Music Cognition and Development, ed. R. Colwell (Oxford: Oxford University Press), 72-123.

Trainor, L. J., McDonald, K. L., and Alain, C. (2002). Automatic and controlled processing of melodic contour and interval information measured by electrical brain activity. J. Cogn. Neurosci. 14, 430-442. doi: 10.1162/089892902317361949

Tremblay, K. L., Piskosz, M., and Souza, P. (2003). Effects of age and age-related hearing loss on the neural representation of speech-cues. Clin. Neurophysiol. 114, 1332-1343. doi: 10.1016/S1388-2457(03)00114-7

Tsjuii, T., Okada, M., and Watanabe, S. (2010). Effects of aging on hemispheric asymmetry in inferior frontal cortex activity during belief-bias syllogistic reasoning: a near-infrared spectroscopy study. Behav. Brain Res. 210, 178-183. doi: 10.1016/j.bbr.2010.02.027

Vermeij, A., van Beek, A. H., Olde Rikkert, M. G., Claassen, J. A., and Kessels, R. P. (2012). Effects of aging on cerebral oxygenation during workingmemory performance: a functional near-infrared spectroscopy study. PLoS ONE 7:e46210. doi: 10.1371/journal.pone.0046210

Vermeire, K., Landsberger, D. M., Van de Heyning, P. H., Voormolen, M., Punte, K. A., Schartzer, R., et al. (2015). Frequency place map for electrical stimulation in cochlear implants: change over time. Hear. Res. 326, 8-14. doi: 10.1016/j.heares.2015.03.011

Wittfoth, M., Schroder, C., Schardt, D. M., Dengler, R., Heinze, H. J., and Kotz, S. A. (2010). On emotional conflict: interference resolution of happy and angry prosody reveals valence specific effects. Cereb. Cortex 20, 383-392. doi: 10.1093/cercor/bhp106

Wong, P. C., Jin, J. X., Gunasekera, G. M., Abel, R., Lee, E. R., and Dhar, S. (2009). Aging and cortical mechanisms of speech perception in noise. Neuropsychologia 47, 693-703. doi: 10.1016/j.neuropsychologia.2008.11.032

Yasumura, A., Inagaki, M., and Hiraki, K. (2014). Relationship between neural activity and executive function: an NIRS study. ISRN Neurosci. 2014:734952. doi: 10.1155/2014/734952

Zatorre, R. J., Belin, P., and Penhune, V. B. (2002). Structure and function of auditory cortex: music and speech. Trends Cog. Sci. 6, 37-46. doi: 10.1016/S1364-6613(00)01816-7

Zhu, M., Chen, B., Galvin, J. J., and Fu, Q. J. (2011). Influence of pitch, timbre and timing cues on melodic contour identification with a competing masker. J. Acoust. Soc. Am. 130, 3562-3565. doi: 10.1121/1.3658474

Conflict of Interest Statement: The authors declare that the research was conducted in the absence of any commercial or financial relationships that could be construed as a potential conflict of interest.

Copyright (C) 2016 Jeong and Ryu. This is an open-access article distributed under the terms of the Creative Commons Attribution License (CC BY). The use, distribution or reproduction in other forums is permitted, provided the original author(s) or licensor are credited and that the original publication in this journal is cited, in accordance with accepted academic practice. No use, distribution or reproduction is permitted which does not comply with these terms. 